\title{
Comparative study on point and line thermographic inspection for fiber orientation assessment of randomly oriented strand material
}

\author{
Henrique Fernandes ${ }^{1 *}$ (D) Hai Zhang ${ }^{2}$, Alisson A.A. Figueiredo ${ }^{3}$, Fernando Malheiros ${ }^{4}$, \\ Luis Henrique Ignaicio ${ }^{5}$, Clemente Ibarra-Castanedo ${ }^{2}$ and Xavier Maldague ${ }^{2}$
}

\begin{abstract}
Composite materials are being successfully used in aeronautics for more than two decades. This great success is mainly because this kind of materials is typically lighter when compared to metals and more resistant to corrosion. Resistance and stiffness of composite materials are usually related to their fiber orientation. The development of non-destructive methods is required to assess this kind of property without damaging the sample. In this work, a non-destructive method based on infrared thermography is presented for fiber orientation assessment. More specifically, two approaches are presented and results are compared for fiber orientation assessment of randomly oriented strand parts. The first one is a point-by-point inspection based on pulsed thermal ellipsometry and the second one is a line approach based on flying laser spot inspection combined with neural artificial networks. Results showed that the second approach provides similar accuracy when compared to the first one; however, it is much faster which makes the second approach very interesting for industrial applications.
\end{abstract}

Keywords: Infrared thermography, Artificial neural networks, Composite materials, Fiber orientation

\section{Introduction}

The use of composite materials reinforced with fibers in astronautics, aeronautics, automotive, wind energy, oil and gas, and petrochemical industries is vital for the full development of these key areas for economic and social prosperity of a country mainly because of composite materials' unique and advantageous properties: these materials are typically lighter and more resistant to corrosion than metallic materials that have traditionally been used in these industries. Techniques and methodologies to ensure the quality of parts, components, and structures made with such class of advanced materials, both in the manufacturing stage and throughout its lifetime, are indispensable, and, towards this strategy and approach, non-destructive evaluation (NDE) methods play today a

*Correspondence: henrique.fernandes@ufu.br

'School of Computer Science, Federal University of Uberlandia, 2121 Joao Naves de Avila Avenue, Uberlandia 38408-100, Brazil

Full list of author information is available at the end of the article fundamental role. NDE has been defined as comprising those methods used to examine or inspect a part or a system without impairing its future usefulness. Many works have recently dealt with such methods [1-4].

Infrared thermography (IRT) is a safe NDE technique that has a fast inspection rate and is generally contactless. It is used for diagnostics and monitoring in several fields such as electrical components, thermal comfort, buildings, artworks, and composite materials. IRT popularity has grown in the recent years due to spatial resolution and acquisition rate improvements of infrared (IR) cameras while they became more affordable. Another factor is the development of advanced image processing techniques focused on this kind of images. In active IRT, an external heat source is used to stimulate the material being inspected in order to generate a thermal contrast between the feature of interest and the background. The active approach is adopted in many cases given that the inspected parts are usually in equilibrium with the surroundings [5]. 
The arrangement or orientation of the fibers relative to one another, the fiber concentration, and the distribution all have a significant influence on the strength and other properties of fiber-reinforced composites. Thus, effective testing techniques are needed to assess fiber content. Destructive methods can be employed to evaluate a composite fiber content, e.g., cutting a section of the material, polishing the area, and evaluating it by microscopy. However, the destructive approach is not always an option since the sample will be "damaged" after the inspection and probably unfit for use. Thus, NDE techniques must be employed in some cases to assess the material's fiber content.

In this paper, we presented an extension of the work presented in [6]. Here, two approaches based on infrared thermography are presented and compared for fiber orientation assessment of randomly oriented strand (ROS) material. The first uses the traditional pulsed thermal ellipsometry (PTE) method which is based on a single heating spot. The second one is based on a recently proposed approach which uses a line heating region produced by a flying laser spot (FLS) and artificial neural networks (ANNs). In this new paper, results of both techniques are presented, compared, and discussed while in [6] only the line (FLS/ANN) is presented and discussed.

\section{Methodology}

\section{Pulsed thermal ellipsometry}

More than one century ago, De Senarmont [7] applied a thermal approach to find out the principal orientations in crystal plates: he covered them with a thin layer of wax, heated them over a small spot, and monitored the isotherm shape revealed by the solid/liquid transition contour appearing in the wax layer. The isotherm proved to be elliptical and its aspect ratio is related to the square root of the principal conductivities in the surface plane.

This method, referred by Krapez [8] as thermal ellipsometry (TE), was later used, with, of course, up-to-date experimental equipment, in several application by the means of IRT [8-10]. It was applied on polymer materials to establish a correlation between their draw ratio and the induced thermal anisotropy. It was also used to evaluate the fiber orientation in the case of composite materials using short or long carbon fibers. In our work, we refer to this method of evaluating the fiber orientation in the case of composite materials (CM) as pulsed thermal ellipsometry-PTE.

PTE is an inspection technique that enables the assessment of fiber orientation on CM. It involves the spot heating of the sample's surface and following the observation of the heated pattern in the IR spectrum. A short pulse is used to spot heat the sample. If the material has an oriented structure, such as carbon fiber-reinforced polymers (CFRP), an elliptical thermal pattern is observed, with the ratio between the two principal axes $(b / a)$ being related to the square root of the thermal diffusivities in the longitudinal and transverse directions. A test on an isotropic material would give a circle instead of an ellipse. Figure 1 shows a typical PTE experimental setup.

The ellipse's major axis, $b$, has the same orientation as the fibers on that region. This "elliptical" behavior occurs due to the difference in the thermal conductivity values on the surface of the sample. The thermal conductivity value parallel to the fibers is greater than the value perpendicular to them, i.e., material is thermally anisotropic:

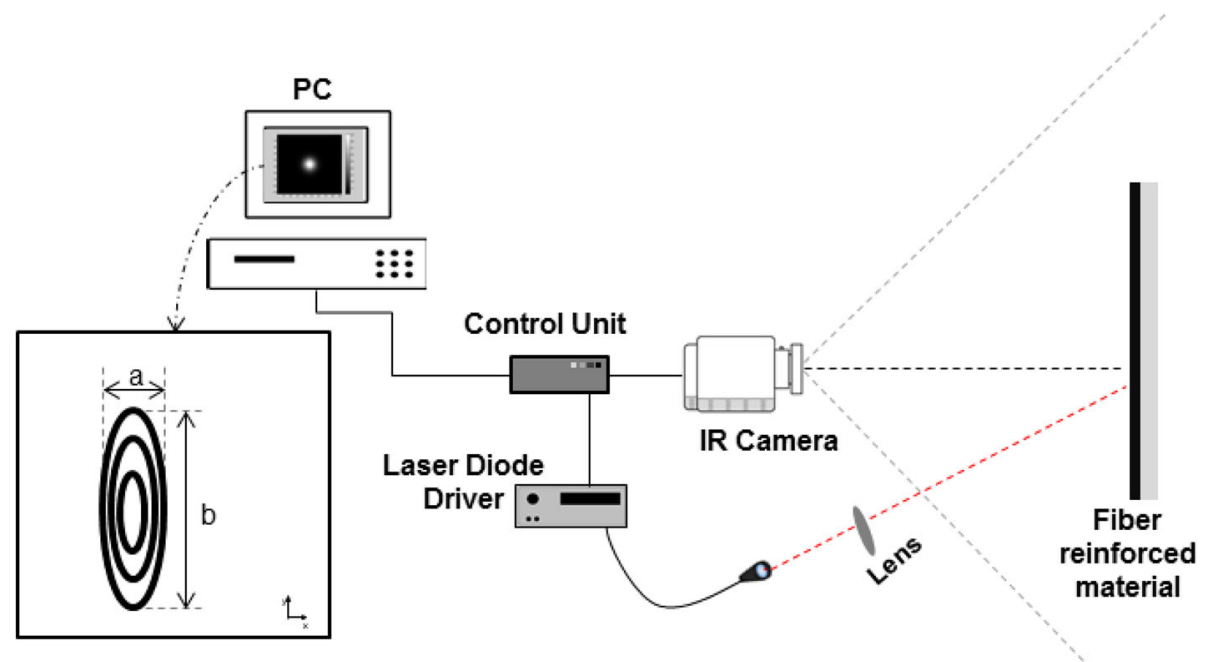

Fig. 1 PTE setup. Pulsed thermal ellipsometry (PTE) setup. Adapted from [11]

C 
$k_{\|}>k_{\perp}$. Thus, heat will travel faster on the direction parallel to the fibers and consequently the thermal pattern observed on the surface will be larger on the direction of $k_{\|}$, which results in an elliptical pattern. The heat diffusion process is recorded with an IR camera and stored in a 3D matrix (or an array of images). In order to extract the elliptical pattern from the IR images, i.e., thermograms, some image processing techniques must be applied. This approach is fully presented in our previous work [11]. In [11], PTE was successfully applied for laminates with continuous fibers. In this kind of materials, fiber orientation is homogeneous. Thus, one single-point heating source inspection is sufficient to assess the material's fiber orientation. On the other hand, for ROS samples, where fiber orientation is randomly distributed on the material's surface, one would need to inspect several points in order to have a good assessment of the material's fiber orientation and distribution. Consequently, such approach would prove very time consuming and unfit for industrial application.

Computational model A computational model is used to simulate the behavior of the heat dissipation on the material without the need of performing an actual experiment. In the current project, computational models were used to provide insights on how the heat would propagate in the sample considering different shapes of heating sources in order to develop the line approach.

In the case of this paper, the phenomenon that is being modeled is transient conduction of heat in solids. This phenomenon can be represented by the heat equation (in this case in the 3D space) which is a parabolic partial differential equation that describes the distribution of heat (or variation in temperature) in a given region over time:

$$
k_{\|} \frac{\partial^{2} T_{s}}{\partial x^{2}}+k_{\perp} \frac{\partial^{2} T_{s}}{\partial y^{2}}+k_{\perp} \frac{\partial^{2} T_{s}}{\partial z^{2}}-\rho C_{p} \frac{\partial T_{s}}{\partial t}=Q
$$

where $k_{\|}[\mathrm{W} / \mathrm{mK}]$ and $k_{\perp}[\mathrm{W} / \mathrm{mK}]$ are the thermal conductivity values parallel and perpendicular to the fibers, respectively; $\rho\left[\mathrm{kg} / \mathrm{m}^{3}\right]$ is the density; $C_{p}[\mathrm{~J} / \mathrm{kgK}]$ is the specific heat of the material; and $Q\left[\mathrm{~W} / \mathrm{m}^{3}\right]$ is the heat source intensity.

A classic method called finite element analysis (FEA) is used to solve Eq. 1. It is a numerical technique that basically works dividing the object's geometry in small sections (called mesh) and finding approximate solutions for the differential equation for each one of these little sections. In the scope of this paper, a commercially available software called COMSOL Multiphysics ${ }^{\circledR}$ was used. COMSOL is a FEA solver and simulation software package for solving various physics and engineering applications. The core package and the heat transfer package are used in our simulations.
The simulation performed was a 0.05 -s laser pulse on a block of a carbon fiber composite which is used with the intent of mimicking the inspection of a single ROS strand. The material's block was carbon/PEEK: a prepreg which $62 \%$ of its weight is AS4 carbon fiber. Thermal properties involved in the simulation are presented in Table 1 . The body heat load within the composite block is given by the following expression [12]:

$$
Q(x, y, z)=Q_{0}\left(1-R_{c}\right) \frac{A_{c}}{\pi \sigma_{x} \sigma_{y}} e^{-\left[\frac{\left(x-x_{0}\right)^{2}}{2 \sigma_{x}^{2}}+\frac{\left(y-y_{0}\right)^{2}}{2 \sigma_{y}^{2}}\right] e^{-A_{c} z}}
$$

where $Q_{0}$ is the total power input, $R_{c}$ is the reflection coefficient, $e^{-\left[\frac{\left(x-x_{0}\right)^{2}}{2 \sigma_{x}^{2}}+\frac{\left(y-y_{0}\right)^{2}}{2 \sigma_{y}^{2}}\right]}$ is the 2D Gaussian distribution in $x y$-plane and $e^{-A_{c} z}$ is the exponential decay due to absorption. The reflection and absorption are assumed to be constants. The planar surface of the composite block incident to the laser beam is assumed to be aligned with the $x y$-plane of the global coordinate system. The top planar surface is aligned with $z=0$. Hence, the effect of absorption can be simulated by the term $\exp \left(-A_{c} *\right.$ $a b s(z))$. The center of the beam is given by the terms $x_{0}$ and $y_{0}$. The beam width and astigmatism are controlled by the standard deviation parameters $\sigma_{x}$ and $\sigma_{y}$.

Infrared image processing Figure 2 summarizes the processing steps involved on the extraction of the fiber orientation based on the data provided by a PTE experiment. The process is fully detailed and discussed in our previous work [11]. Nonetheless, their major points are going to be outlined here.

Optimal diffusion time selection As stated in beginning of this subsection, a heating spot, produced by a laser beam, is used to stimulate the surface of the sample producing the thermal behavior that will lead to the fiber orientation assessment. After the spot is heated, the heat diffusion process is recorded with an IR camera and stored in a 3D matrix $M$, i.e., an array of images, for postprocessing. The size of $M$ is $m \times n \times k$ which means that it is composed of $k$ images of size $m \times n$. The number of images, or thermograms, depends on the duration of the acquisition as well as the camera's acquisition frame rate.

Table 1 Thermal properties of carbon/PEEK [24]

\begin{tabular}{llll}
\hline & Property & Value \\
\hline$k:$ & Thermal conductivity & Parallel & $5.65[\mathrm{~W} / \mathrm{mK}]$ \\
& & Perpendicular & $0.35[\mathrm{~W} / \mathrm{mK}]$ \\
$C_{p}:$ & Specific heat ${ }^{a}$ & $1310[\mathrm{~J} / \mathrm{kgK}]$ \\
$\rho:$ & Density & $1584\left[\mathrm{~kg} / \mathrm{m}^{3}\right]$ \\
\hline${ }^{{ }^{a} \text { At constant pressure }}$ & &
\end{tabular}




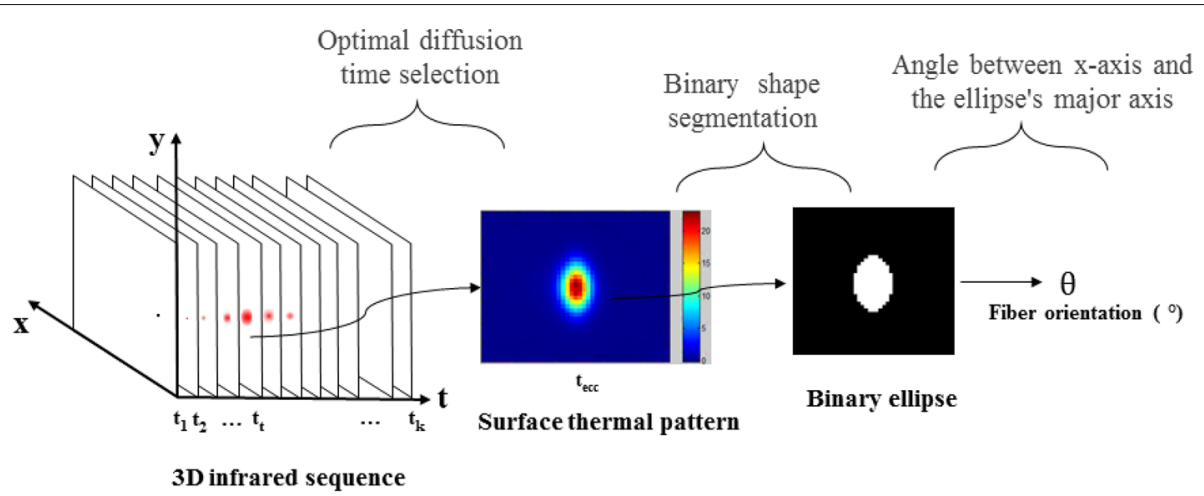

Fig. 2 Processing steps. Infrared image processing steps for PTE. Adapted from [11]

This sequence of images contains the following: the plate before heating, the moment when the beam heated the plate, the rise of the temperature profile, and finally the temperature profile decrease. In the case of our ROS samples, which are thermally anisotropic, the heat pulse will produce an elliptical pattern on the surface of the sample. However, this pattern would be closer to a circle on the very beginning of the experiment and in later times where the thermal behavior of deeper layers would be much more present. Thus, our assumption is that sometime between these two moments, an image would contain the best pattern that reflects the fiber orientation on the surface, i.e., the direction in which the thermal conductivity is higher. This optimal moment is selected by calculating the eccentricity $(\varepsilon)$ of each elliptical thermal pattern on the thermograms on $M$ and choosing the image which contains the ellipse with the largest $\varepsilon$. Eccentricity $(\varepsilon)$ is a quantity defined for a conic section which can be given in terms of major and minor axes. In the case of a circle $\varepsilon=0$ while in the case of an ellipse $0<\varepsilon<1$. Based on this idea, the thermal pattern that would better express the fiber orientation is the one with the largest $\varepsilon$. During our experiments, it always occurred before $0.5 \mathrm{~s}$ after the beam has been stopped.

Binary shape segmentation After the image with the optimal diffusion time was selected, the next step is to segment, or binarize, the ellipse on the image. This is a simple task that can be accomplished by using the automatic clustering-based image thresholding method. There is a wide range of classical histogram-based algorithms for automatic threshold selection for bimodal and multimodal images available in the literature. In this work, Otsu's method [13], a well-established method, is used.

After the selection of the threshold level, the binary image is created by applying the following characteristic function in the infrared image selected based on the optimal diffusion time calculated in the previous step:

$$
f b e\left(P_{T i}\right)= \begin{cases}1, & \text { if } T_{i} \geq T h \\ 0, & \text { otherwise }\end{cases}
$$

where $i \in[1, \ldots, m \times n], m \times n$ is the number of pixels in the image and $T h$ is the temperature threshold value determined by Otsu's method.

Calculation of angle between $x$-axis and binary ellipse's major axis With the resulting binary image obtained via Eq. 3, which is represented by a binary matrix, a simple Matlab $^{\odot}$ native function was used to calculate the ellipse orientation. The orientation of the image, here the binary ellipse, is the angle (in degrees ranging from $-90^{\circ}$ to $90^{\circ}$ ) between the $x$-axis and the major axis of the ellipse that has the same secondmoments as the region.

\section{Line inspection approach}

A second inspection approach is used to produce a heating line on the surface of the samples in order to enable the inspection of a larger area at once. It is based on a dynamic point scanning approach. The use of a flying laser spot (FLS) technique in combination with an artificial neural network was proposed in our previous work [14] to assess the fiber orientation over a line region on the surface of a ROS plate. In this paper, we conduct a brief review of the approach and present additional and unpublished results.

Flying laser spot FLS is a dynamic active IRT technique, which can be employed for the inspection of materials by heating point-by-point a component while acquiring a series of images using an IR camera. This can be done in two ways, either the thermographic head, consisting of an IR camera and an energy source, moves along the surface while the sample to be inspected is motionless, or it may 
be the sample that is in motion while the thermographic head stands still. In both cases, the thermal history for every pixel can be precisely tracked by controlling the displacement speed, either the laser or the sample, and the rate of data acquisition. Detailed theoretical and experimental aspects of this technique can be found in [15]. In this paper, the second displacement approach is adopted.

In the recorded IR raw sequence, the sample undoubtedly appears to be moving. In order to perform advanced image process techniques, the sequence must be rearranged into a pseudo-static sequence so that the sample appears motionless. The reconstructed sequence is obtained by following the temporal evolution of every pixel independently, in such a way that a given pixel of the original sequence $P\left(x_{i}, y_{j}, t\right)$ is recovered frame by frame through time $t$ and reallocated into a new image. For instance, a pixel $P$ that is in a given position at time $t$ will be in a different position at a later time. Figure 3 shows some images of an original sequence and an image from the corresponding pseudo-static reconstructed sequence $0.37 \mathrm{~s}$ after the pulse. Part of the experimental setup used is also shown (the laser source is not shown). The arrow indicates the direction of the sample's movement. A detailed procedure on how to reconstruct the dynamic sequence in order to obtain a pseudo-static sequence is provided in [16].

After the pseudo-static sequence is obtained, some advanced infrared image processing technique must be applied in the reconstructed sequence in order to perform some feature selection to be used in the ANN step. In this work, three processing techniques were tested: principal component thermography (PCT), pulsed phase thermography (PPT), and dynamic thermal tomography (DTT). PCT, originally proposed in [17], extracts the image features and reduces undesirable noise. In PPT, originally proposed by [18], data is transformed from the time domain to the frequency spectra. The phase is very attractive not only for qualitative inspections but also for quantitative characterization of materials. In DTT, originally proposed by [19], pixel temperature profiles are rearranged so the time when the maximum temperature occurred for each pixel can be displayed in a single image. Details of this infrared image processing techniques are omitted here; nonetheless, they can be easily found in the literature.

Artificial neural network An ANN is an information processing paradigm that is inspired by the way biological nervous systems, such as the brain, process information. It is composed of a large number of highly interconnected processing elements (neurons) working in unison to solve specific problems. ANNs, like people, learn by example. An ANN is configured for a specific application, such as pattern recognition or data classification, through a learning process which could be supervised or unsupervised. Learning in biological systems involves adjustments to the synaptic connections that exist between the neurons. This is true of ANNs as well. There are several works involving ANN in the literature. A review on image processing with ANN can be found in [20]. In IRT, ANN has also been used for years for defect depth estimation [21, 22].

In this work, the thermal pattern obtained from the pseudo-static sequence is divided into small sections (or samples) that represent different points on the inspected

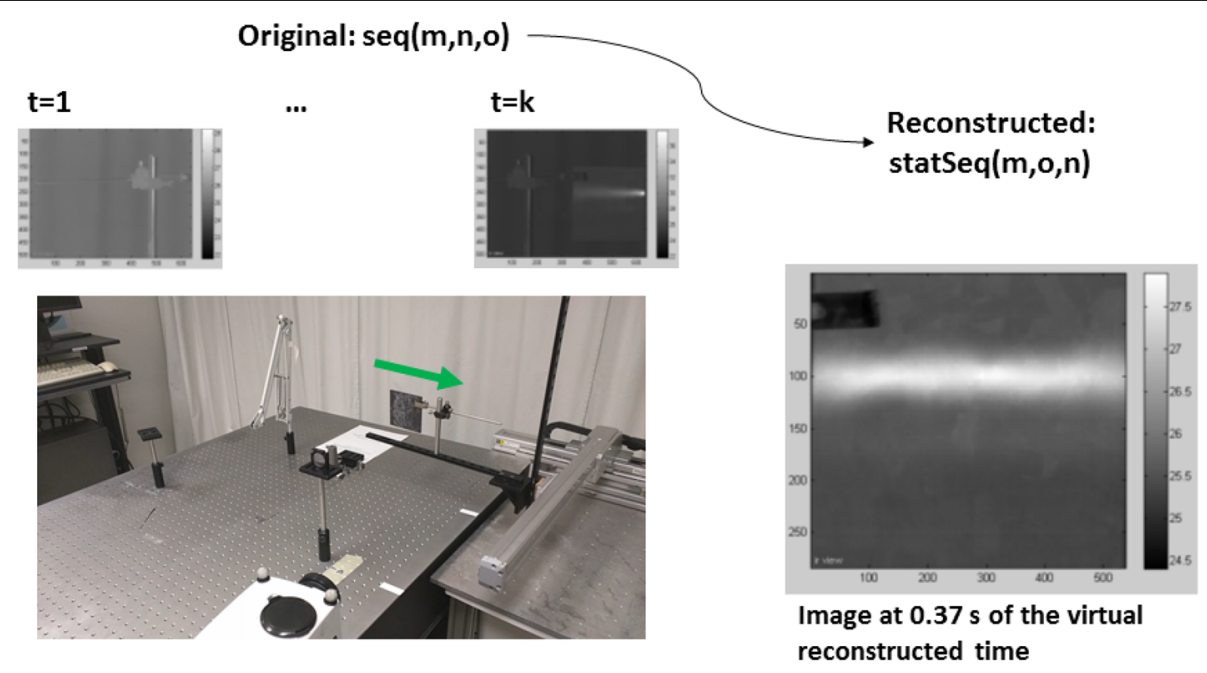

Fig. 3 Pseudo-static sequence reconstruction. In the top of the figure, two images from the original sequence at different times are shown. Below these images, the experimental setup is shown: the IR camera and the inspected sample can be seen as well as the 2-axis electric actuator used to displace the sample. The green arrow indicates the direction of displacement. On the right, an image from the reconstructed pseudo-static sequence is shown. Adapted from [14] 
line. A two-layer feed-forward network, with sigmoid hidden and softmax output neurons (512 and 4, respectively) trained with scaled conjugate gradient backpropagation, is then used to classify these points on the reconstructed line obtained with the pseudo-static IR sequence into their corresponding class (in this case: fiber orientation). The points (samples) extracted from the pseudo-static line are divided into three sets of samples: $70 \%$ of the total of samples is used to train the network, $15 \%$ is used for validation during training, and the remaining $15 \%$ is used for testing the network after the training has been completed. Next, data representation of input samples is detailed as well as how classes are organized.

After the thermal pattern obtained with the line approach (FLS) was reconstructed into the pseudo-static sequence, the resulting image sequence is processed with one of the three techniques mentioned before: PCT, PPT, or DTT. Then, the processed image is binarized using an automatic threshold selection based on Otsu's method [13]. The envelope of the binary line image is easily selected and then 11 features are calculated for each sample. Figure 4 shows the binary images obtained from the processed reconstructed lines. From these binary images, an envelope is extracted. Each sample has two line segments originating from the line envelope: top and bottom line segments. These line segments belong to the edges of the binary image. The first two extracted features are the normal to the line segment orientation regarding the $x$-axis: $\theta_{1}$ and $\theta_{2}$. The next two features are the curvature values $\left(k_{1}\right.$ and $k_{2}$ : it is a measure of how much the curve deviates from a straight line) in the middle points of the top and bottom line segments. The fifth feature is the width of the envelope on its middle section. The last six extracted features are also related to the width of the envelop: the distance (in pixels) from the envelope's centroid to six points on the envelope edges.

The processed pseudo-static image is divided in the same manner that the binary image was divided. Then, the pixels of each sample are rearranged line-wise. The input data of the ANN, i.e., the data used to describe each sample, is the combination of the 11 features calculated before, with the line-wise pixels from the first empirical orthogonal function (EOF) for the PCT case, the phase image for the PPT case, and the resulting image for the DTT case. In total, each sample is represented by a set of 2681 numerical values. These values are the input of the network.

The output of the ANN is the class of the sample presented in the input layer. In this work, samples were classified into four classes. Each class covers a range of $45^{\circ}$. For instance, class 1 (blue) is centered at $0^{\circ}$ and represents orientation angles ranging from $157.5^{\circ}$ to $22.5^{\circ}$, class 2 (green) is centered at $45^{\circ}$ and represents orientation angles ranging from $22.5^{\circ}$ to $67.5^{\circ}$, class 3 (orange) is centered at $135^{\circ}$ and represents orientation angles ranging from $157.5^{\circ}$ to $112.5^{\circ}$, and class 4 (red) is centered at $90^{\circ}$ and represents orientation angles ranging from $67.5^{\circ}$ to $112.5^{\circ}$. Thus, the output layer of the ANN has four neurons. Each neuron is in charge of recognizing one class, i.e., if the sample presented in the input layer belongs to the first class, the first neuron would have the value 1 and the other neurons' value would be 0 (in the perfect recognition scenario).

In order to train the network, target classes were previously determined by PTE static-point inspections. For each one of the 49 samples on the line, a respective PTE inspection was previously performed, i.e., a pointby-point inspection: for each line, 49 samples, with 2-mm spacing, were inspected with the PTE approach proposed in [11]. Then, based on the orientation angle obtained with the PTE inspection, a target class was assigned to the sample. All 180 angle degrees possibilities were not considered as individual classes because it would make the classification processes impossible due to the lack of information present on the line envelope that could be extracted. For instance, the distinction between an input belonging to class $15^{\circ}$ and an input belonging to class $16^{\circ}$ would be impossible.

\section{Experimental setup and inspected samples PTE setup}

Two ROS samples were inspected for the PTE approach. The first ROS flat plate, ROS001 (figure not shown here), is a $250 \times 150-\mathrm{mm}$ plate molded using carbon/PEEK unidirectional slit tape, which was cut into strands of $25.4 \times 6.35 \mathrm{~mm}$ using an automated tape cutter. The other ROS sample, B61 (Fig. 5), is a $100 \times 100$-mm plate molded also using carbon/PEEK unidirectional slit tape, which was cut into strands of $25 \times 10 \mathrm{~mm}$ using an automated tape cutter. Figure $5 \mathrm{a}$ shows the front side of the plate and Fig. $5 \mathrm{~b}$ shows the back side of the plate.

The samples were inspected by PTE using a laser-diode beam spot as stimulation source. A computer and a control unit were used to store recorded images and control the laser driver as well as the IR camera. A high-power laser diode available in our laboratory was used in the experiments. Its output power goes from 0 to $30 \mathrm{~W}$ and its wavelength is $808 \pm 5 \mathrm{~nm}$. A biconvex lens was used to focus the beam on a small spot on the surface of the sample. Finally, a mid-wave infrared camera (MWRI) - Indigo Phoenix Thermal Camera from FLIR Systems ${ }^{\odot}$, InSb, 3$5 \mu \mathrm{m}, 640 \times 512$ pixels windowed to $320 \times 256$ pixels at a frame rate of $220 \mathrm{~Hz}$ was used to record the temperature profiles.

For our first round of experiments, sample ROS001 was placed in front of the focused laser beam. The angle of incidence (AOI) of the laser beam with the plate's surface, i.e., the angle between the incident beam on the surface 


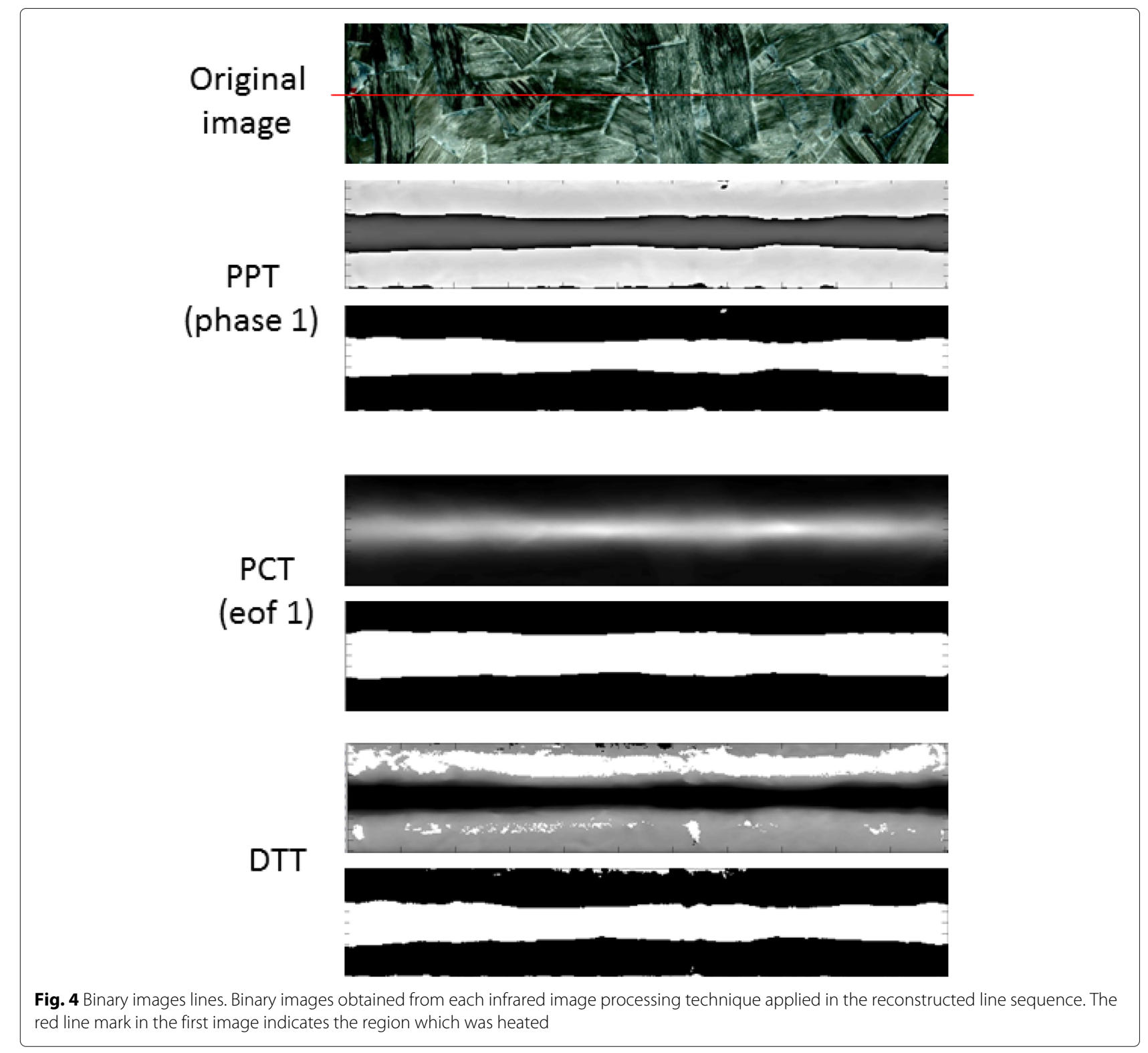

and the line perpendicular to the surface at the point of incidence, was $0^{\circ}$ while the camera's optical axis AOI was about $15^{\circ}$, i.e., the angle between the camera optical axis and the plate's normal to the surface.

A short pulse of $0.1 \mathrm{~s}$ was shot heating a small circle area on the plate's surface. Then, the heating and cooling down process was recorded using the MWIR camera. As mentioned before, the pattern formed on the plate's surface is elliptical in anisotropic materials, which is the case of our samples and the ellipse major axis is related to the fiber orientation on the surface. The parameters used in this experiment are listed in Table 2.

In order to assess the fiber orientation of a broader region, a second round of experiments was performed. For this round, a section of each surface of the sample B61 was inspected with a point-by-point approach in order to create an "orientation map." The dimension of each section was $50 \times 50 \mathrm{~mm}^{2}$. Figure 5 shows the inspected sections. The distance between each inspected point was $5 \mathrm{~mm}$. This 5 - $\mathrm{mm}$ distance was chosen so every strand $\left(25 \times 10 \mathrm{~mm}^{2}\right)$ on the section had at least one point heating it. With this configuration, 121 points were inspected on each section. The other inspection parameters were kept the same as the ones used in the previous experiment and are listed in Table 2. The inspection of each section, i.e., 121 points, took about $2 \mathrm{~h}$.

The horizontal displacement of the sample was performed with an aid of a 2-axis electric actuator, which moves the inspected sample from left to right (or right to left) in front of the camera's field of view. The inspection of 


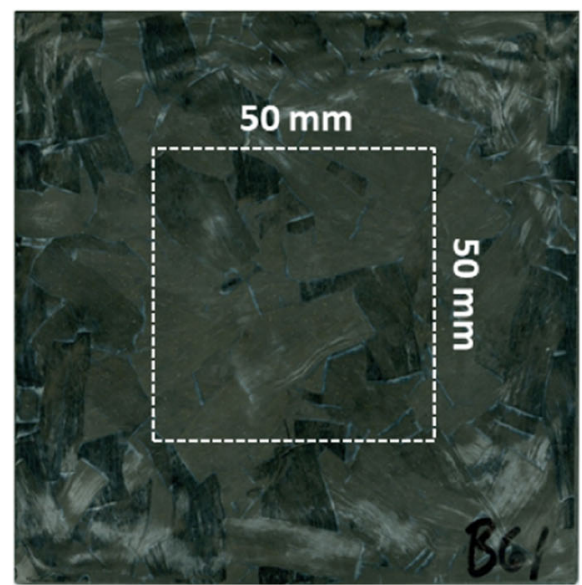

a

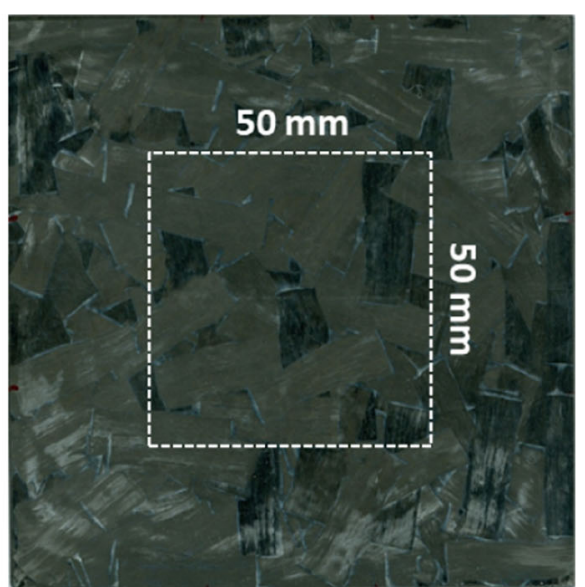

b

Fig. 5 Second ROS sample (B61). Second inspected ROS sample with the regions inspected on the surfaces of sample depicted with the dashed square. a Front surface and $\mathbf{b}$ back surface

a complete row (with 11 inspections) was performed, and then, the sample was manually repositioned so the second row, $5 \mathrm{~mm}$ below, could be inspected. Figure 6 shows the experimental setup used during this inspections. On the left, an image with front surface of the sample B61 is shown. The red point indicates the location of the first point of the first row. On the right, the experimental setup is shown with the electric actuator, the IR camera, and the IR laser diode.

\section{Line inspection approach setup}

The samples inspected with the line approach are similar (same specifications) to the second sample inspected with the PTE approach. The total of three samples were used and four lines $(\mathrm{H} 1, \mathrm{H} 2, \mathrm{~V} 1$, and V2) on each sample were inspected. Thus, 12 lines were inspected from three different samples (B61, B62, and B63). Figure 7 shows these samples with the respective inspected lines indicated with red lines.

For each inspection, the sample to be inspected is displaced in front of the thermographic head (camera and laser). In the first recorded image the sample does not

Table 2 Parameters used during PTE inspections

\begin{tabular}{lll}
\hline Parameter & Value & \\
\hline Diode-laser frequency & 805 & {$[\mathrm{~W} / \mathrm{mk}]$} \\
Diode-laser power & 1 & $\mathrm{~W}$ \\
Shooting duration & 0.1 & $\mathrm{~s}$ \\
Spot size on plate's surface & 2 & $\mathrm{~mm}$ \\
Acquired frames & 1200 & frames \\
Camera's acquisition rate & 220 & $\mathrm{~Hz}$ \\
Camera's frame size & $320 \times 256$ & pixels \\
\hline
\end{tabular}

appear and in the last one the sample does not appear either. In the images between the first and last one, the sample appears in different positions. This displacement of the sample is performed with the aid of a 2-axis electric actuator that moves the inspected sample from left to right (or right to left) in front of the camera's field of view. The use of a 2-axis electric actuator provides the possibility to program the inspection path and to control the inspection speed displacement and acquisition rate in a precise manner. After a line was inspected, the sample was repositioned so the laser spot could head the desired line. This approach was repeated until the 12 lines were inspected. The experimental setup prepared in the laboratory can be seen in Fig. 3. Next, results are presented and discussed.

\section{Results and discussion \\ PTE results}

Computational model Previous to performing the PTE experimental tests, FEA studies were conducted. Computational models were created using the commercial software COMSOL Multiphysics ${ }^{\circledR}$. The first model created investigates the transient heating of a composite material single strand when an incident laser beam in continuous wave $(\mathrm{CW})$ mode shines upon it for a given time. Since the thermal conductivity values in $x$ and in $y$ are different due to the carbon fiber thermal behavior (one is much bigger than the other), an elliptical pattern is expected to be formed on the $x y$-plane. The mesh used in the simulation is showed in Fig. 8 (the scale is in millimeter). An elliptical boundary was created on the top surface to represent the zone of heat input and a finer mesh was used in this region.

In Fig. 9, some plots of the results calculated by the software are shown. Figure 9a shows temperature 


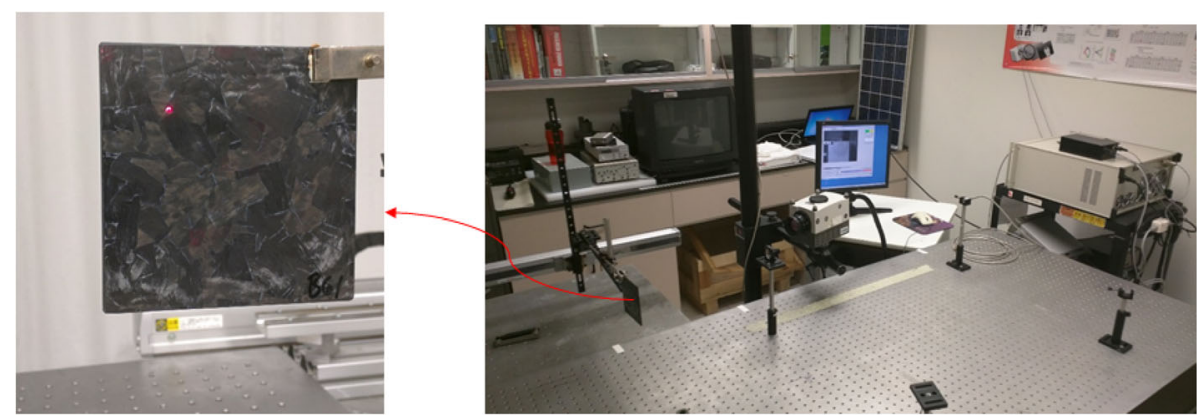

Fig. 6 Point-by-point setup inspection. PTE setup used for the point-by-point inspection. The red point on the image on the left indicates the position of the first inspection on the first row

distribution over the $x y$-plane at time $0.3 \mathrm{~s}$, and Fig. $9 \mathrm{~b}$ shows the temperature counters at the same time. Figure 10 shows the plot of temperature values over the line and the column passing through the point where the beam center was. It is possible to see in both Figs. 9 and
10 that the heat has spread faster in the direction which the thermal conductivity is higher, i.e., in the direction parallel to the fibers.

The second simulation carried out was about heating more than one block, or strand, at the same time. This

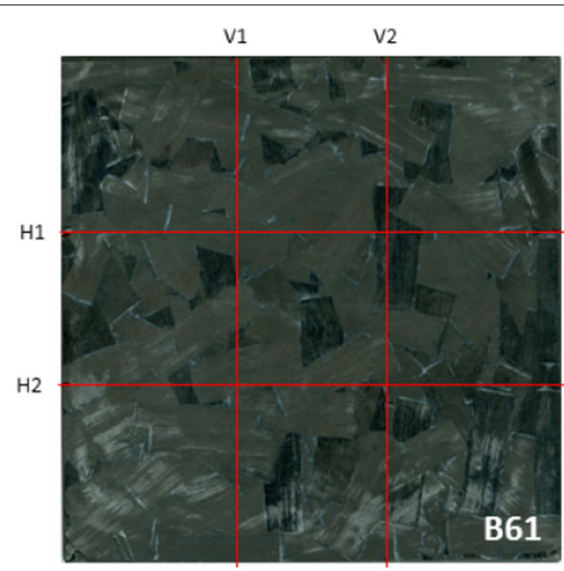

a

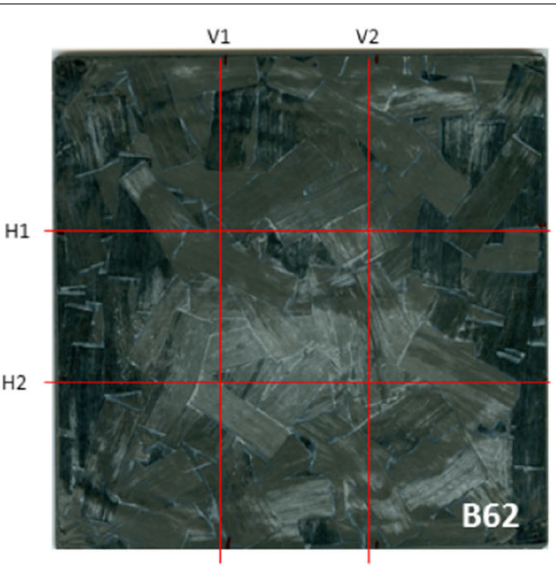

b

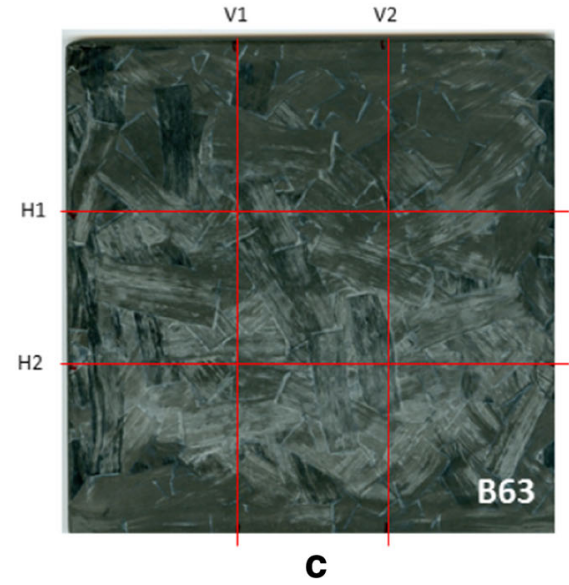

Fig. 7 Inspected sample with the line approach. ROS samples inspected with the line approach. The red lines indicate where the inspected regions were. a $\mathrm{B} 61, \mathbf{b} \mathrm{B} 62$, and $\mathbf{c} \mathrm{B} 63$ 


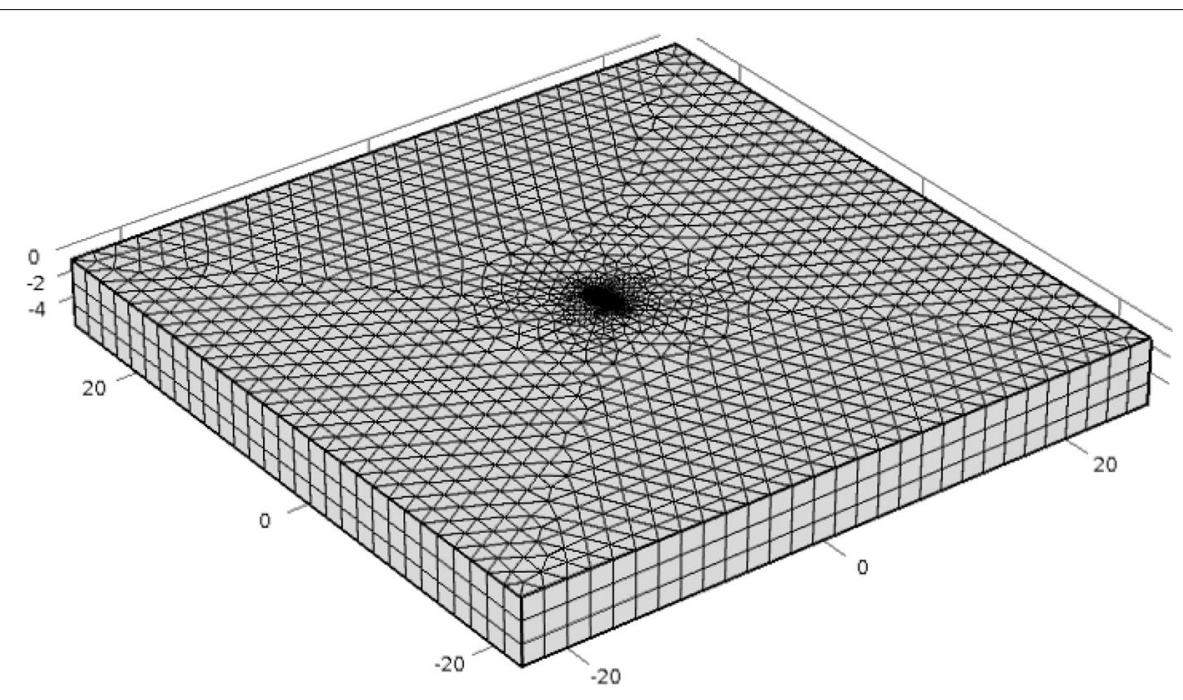

$$
x_{i}^{2}=x
$$

Fig. 8 COMSOL mesh. Mesh used by COMSOL to solve the heat transfer problem

was performed simulating a line heating where the standard deviation parameters, $\sigma_{x}$ and $\sigma_{y}$, were different. One was much bigger than the other. Also, neighbor strands had different thermal conductivities to simulate strands with fibers oriented in $0^{\circ}$ and $90^{\circ}$. The goal of this simulation was to see the different heat patterns formed over the heating line caused by the different thermal conductivities in different regions. The goal is to use such configuration to inspect a bigger area at once in a ROS plate.

Simulation results obtained showed that it is possible to identify different patterns along the line due to strands' different fiber orientation (or thermal conductivity). Thus, these preliminary results show the potential of line heating to inspect a bigger ROS area. Figure 11 shows the isothermal contours obtained at time $0.3 \mathrm{~s}$ for this second simulation.
Using the same geometry used in the second simulation, a third simulation was performed in order to investigate the effect of the size of the heating spot and its position. First, the beam center was positioned exactly between two different strands. It was observed that the heat pattern formed on the surface is deformed in this case. Part of the heat follows the fiber orientation of the first strand while the rest follow the fiber orientation of the second strand resulting in a deformed-two-region ellipse. Figure 12 shows the temperature on the surface at time $0.3 \mathrm{~s}$ for this simulation.

The size of the spot also plays a key role here. Since a single narrow strand is being analyzed at a time, the spot should be small enough that the heat has space to propagate freely and the appropriate thermal partner is observed. For instance, the simulation result illustrated in Fig. 13 shows that if a big spot is used, it is not possible
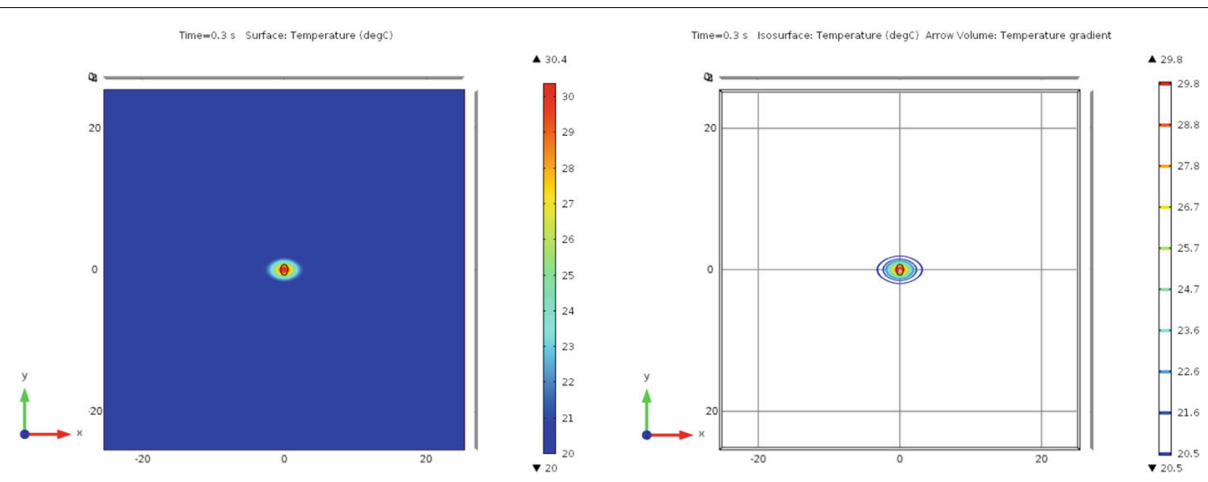

Fig. 9 Simulated temperature at surface. Temperature over $x y$-plane at time $0.3 \mathrm{~s}$ calculated with the simulation 


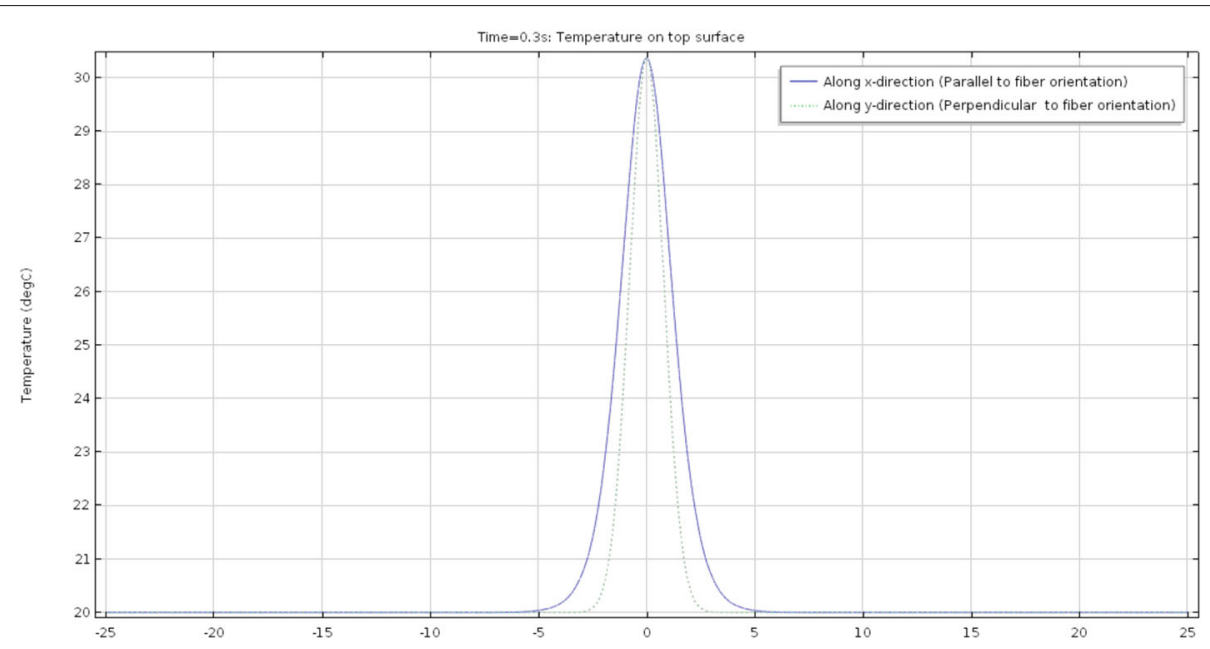

Fig. 10 Surface temperature profile. Temperature distribution on the surface $[\mathrm{mm}]$ over $x$ and $y$ directions (line $x$ column)

to see the fiber orientation. In the example, the fiber orientation of the heated strand is $90^{\circ}$ and the figure shows the temperature distribution at time $0.3 \mathrm{~s}$. By looking at these results, one cannot surely say that the fiber orientation is $0^{\circ}$ or say that it is $90^{\circ}$. Thus, it is important to choose properly the spot size and its position according to the specimens (region) being tested.

Experimental In order to evaluate the effectiveness of the PTE approach for ROS samples, individual strands were first inspected separately. The results obtained for two different strands chosen on the surface of the sample ROS001 are presented here. A first PTE inspection was performed. Next, the plate was rotated $90^{\circ}$ clockwise and a second PTE inspection was then performed. Inspections were expected to be also rotated $90^{\circ}$ from each other.

Figures 14 and 15 show the results of those inspections. Figure 14 shows the results for the first inspected strand. Figure 14a, b shows the results in the case where the plate was in its original position while Fig. 14c, d shows the results in the case where the plate was rotated $90^{\circ}$ clockwise. Figure 15 shows the results for the second inspected strand. Results for both strands are presented in a similar structure. Images on the left column display the picture of the region on the plate containing the specific inspected strand (position where the laser beam heated the surface

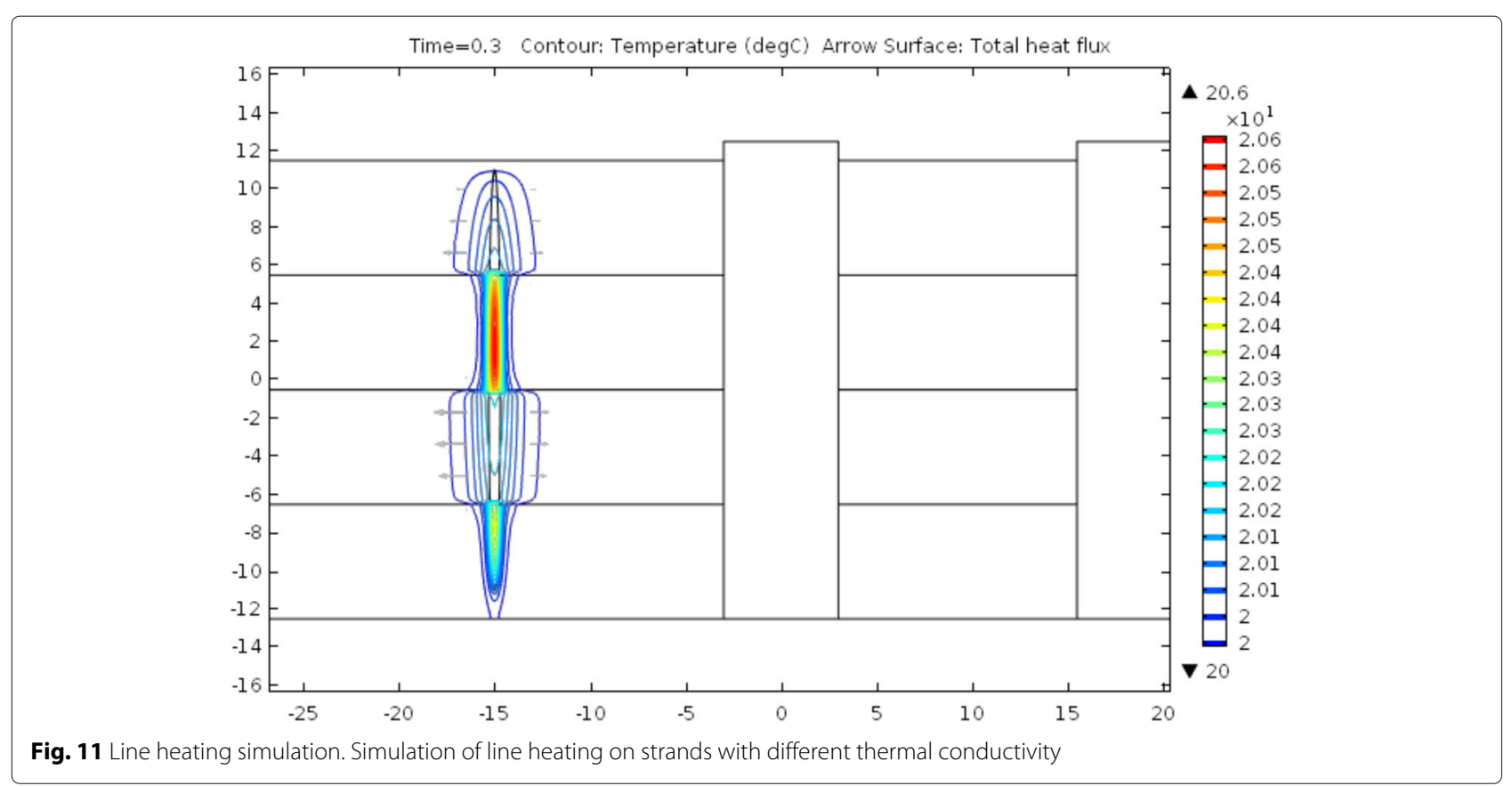




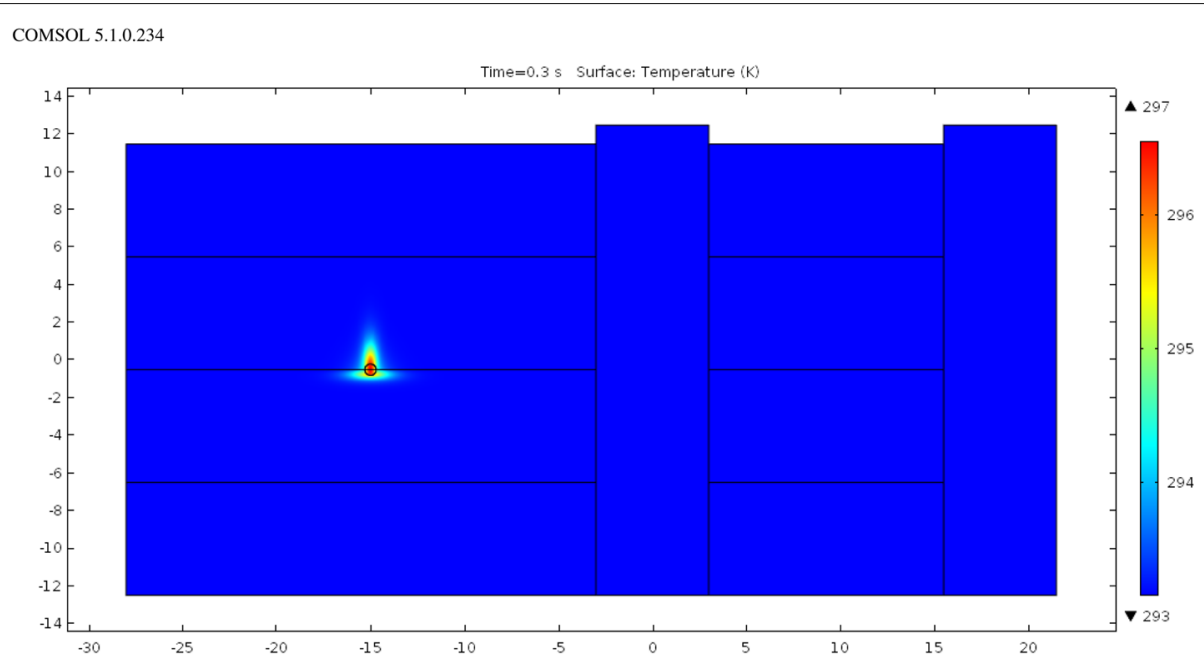

Fig. 12 Simultaneously heating two strands. Simulation of effect of heat dissipation between two different strands at time $0.3 \mathrm{~s}$

is marked with a red point). Images on the right column display the respective ellipse segmented with the approach described in the "Methodology" section (red line indicates the ellipse's major axis).

Fiber orientation measured in Fig. 14b was $-47.52^{\circ}$ while the fiber orientation measured in Fig. 14d was $55.99^{\circ}$. The ellipse extracted from the second inspection is then $76.49^{\circ}$ shifted when it should be $90^{\circ}$. Thus, an error of approximately $13.5^{\circ}$ is present in this case. For the second strand, fiber orientation measured in Fig. 15b was $6.37^{\circ}$ while the fiber orientation measured in Fig. $15 \mathrm{~d}$ was $-85.19^{\circ}$. The ellipse extracted from the second inspection is then $88.5^{\circ}$ shifted when it should be $90^{\circ}$. Thus, an error of approximately $1.5^{\circ}$ is present in this second case. It is important to remember that all fiber orientation angles are reported regarding the $x$-axis.
For both strands, the difference between the two major axis measurements (fiber orientation), i.e., first when the plate was in its original position and then in when it is rotated $90^{\circ}$ clockwise, should be $90^{\circ}$. However, it is not. For the first strand, the error between the two measurements was approximately $13.5^{\circ}$. This happened because the exact position heated in each case was not the same and, perhaps, in the first inspection a place nearer to the chip border was heated and it got more influence from a neighbor strand, which could have a different fiber orientation. Additionally, during the plate's molding process, heat and pressure are applied on the strands, which leads to a high degree of deformation in the shape of consolidate strands in the final plate and it could give to strands a high level of "shape interaction." Thus, in some cases, neighbor strands can play a bigger role in the

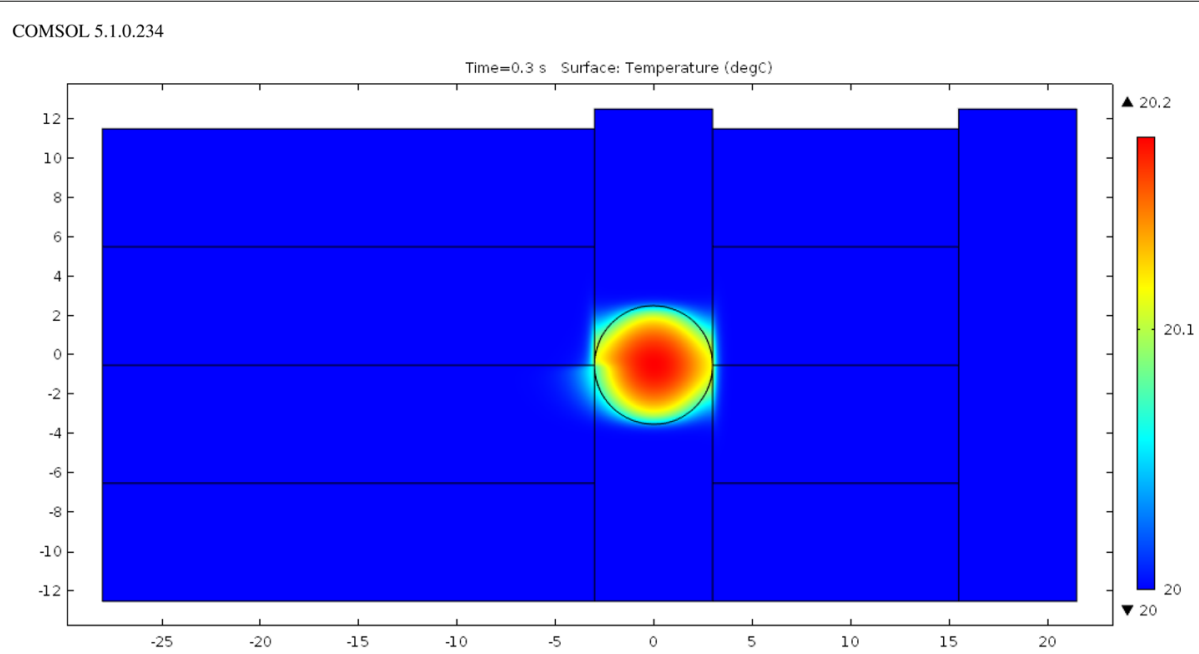

Fig. 13 Strand heat with big spot. COMSOL simulation of effect of spot heating size 


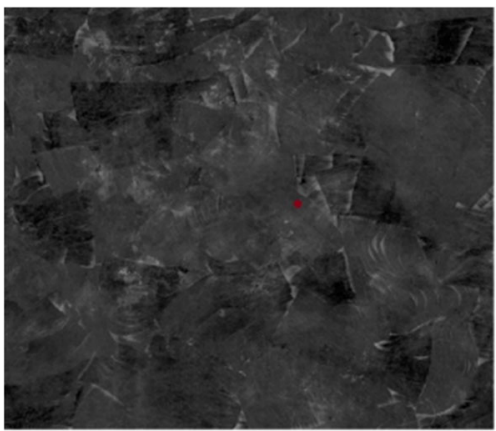

a

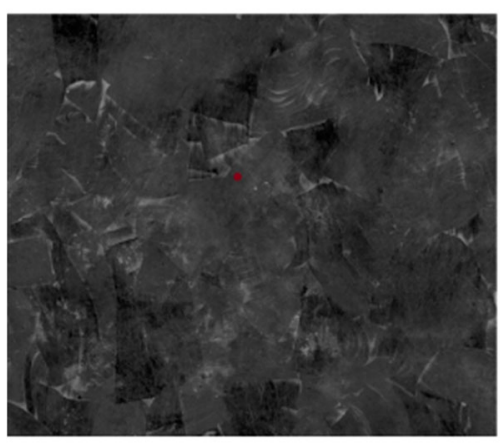

C
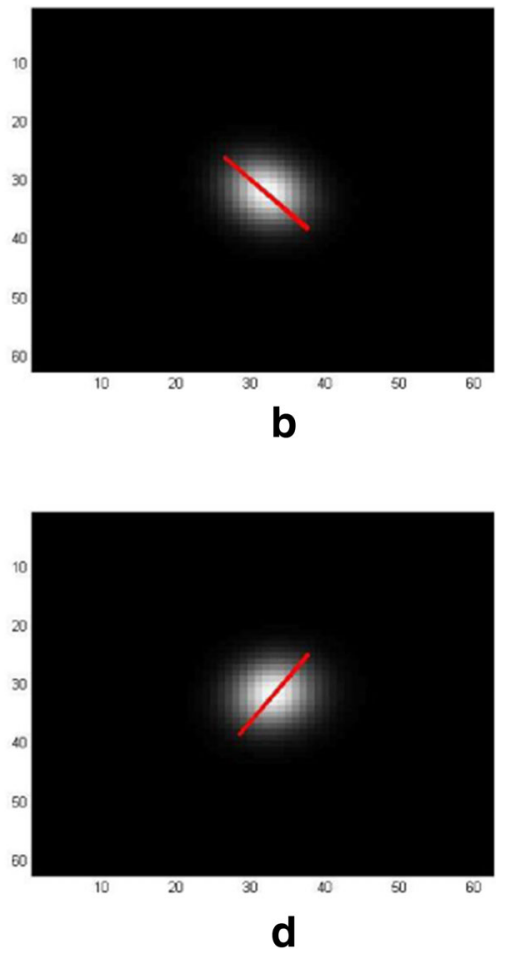

Fig. 14 First PTE inspection of single strand. First strand: $\mathbf{a}$, b plate on its original position and $\mathbf{c}$, $\mathbf{d}$ plate rotated $90^{\circ}$ clockwise

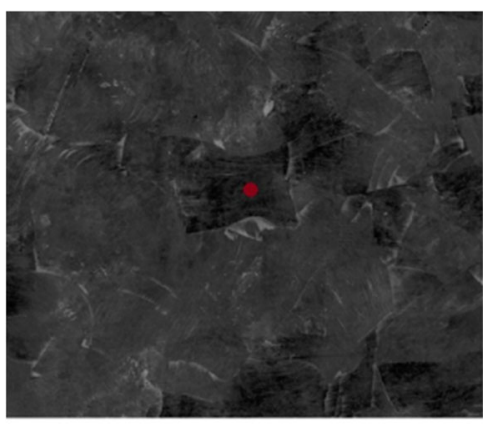

$\mathbf{a}$

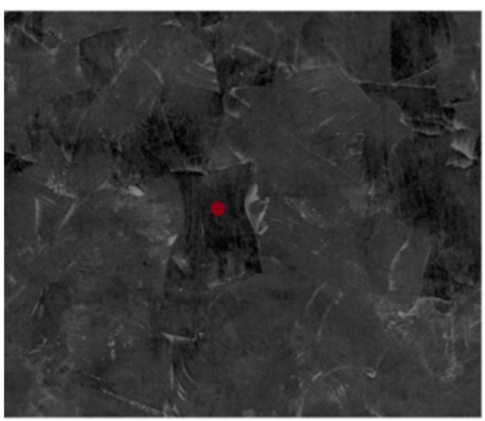

C

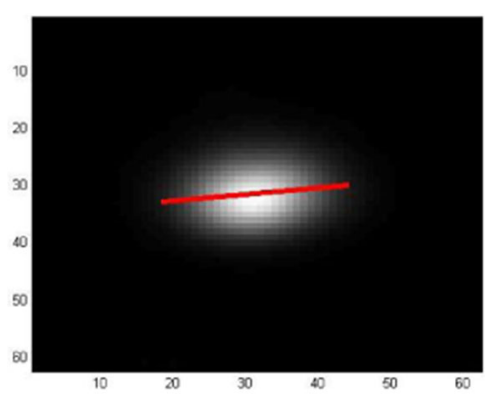

b

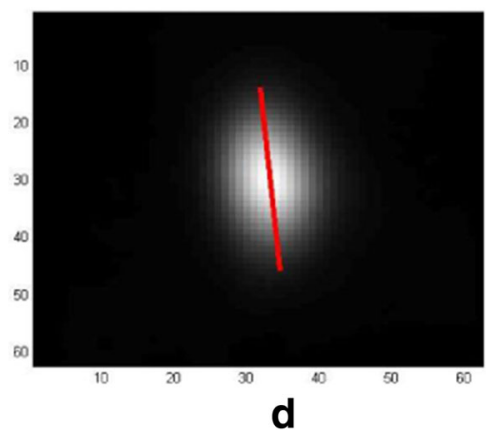

Fig. 15 Second PTE inspection of single strand. Second strand: $\mathbf{a}, \mathbf{b}$ plate on its original position and $\mathbf{c}$, $\mathbf{d}$ plate rotated $90^{\circ} \mathrm{clockwise}$ 


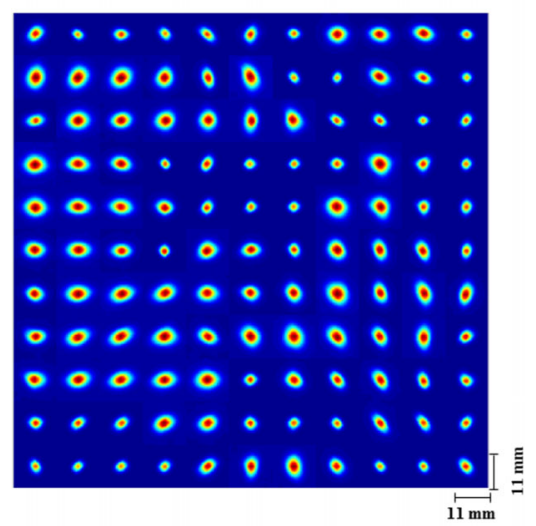

a

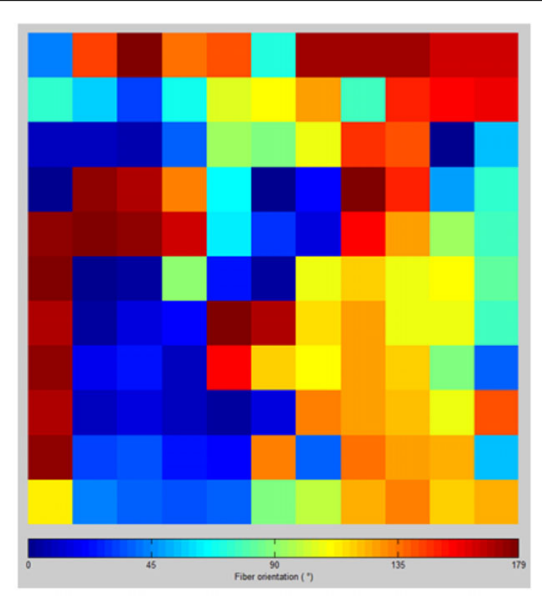

b

Fig. 16 Point-by-point inspection results—front section. Result of point-by-point inspection of the front section of sample B61. a 121 ellipses obtained and $\mathbf{b}$ respective color-coded orientation map. The actual size of the inspected region is $50 \times 50 \mathrm{~mm}^{2}$

heating diffusion on a single strand, which could affect the elliptical pattern. For the second inspected strand, the error between the two inspections was approximately $1.5^{\circ}$. Here the error between measurements is much smaller. This happened because the second strand is better placed and large enough so the heating spot could hit the same position with minimal edge or neighbor strand effects.

Therefore, one can conclude from the computational results and this first experimental results presented that, similar to the laminate case (which were approached in our previous works $[11,23]$ ), where the fiber orientation on each layer of the laminate is uniform, fiber orientation can also be assessed for ROS material using PTE.

So far, it was presented the use of PTE to measure the fiber orientation of a single strand on the surface of a ROS sample. However, only the local inspection of a single strand is not enough to meaningfully assess the fiber orientation on the surface of a ROS sample. In order to have an assessment about how random and how strands (fiber orientation) are distributed, several points, systematically distributed on the surface of the ROS sample, should be inspected. The outcome of such inspection is going to be called "orientation map."

A section of each surface of the sample B61 was inspected with a point-by-point approach in order to create the so-called orientation map. The dimension of each section was $50 \times 50 \mathrm{~mm}^{2}$. A total of 121 points was inspected for each section. Figure 5 shows the inspected sections, and the details of the experimental procedure can be found in the previous section.

The fiber orientation was then calculated for each inspected point according to what was described in the "Methodology" section. Decimal values of each measured angle were not considered and the angle values were rounded. Thus, the fiber orientation assessed on each point was reported in one of 180 possible angles. In order to create the map, each possible angle was assigned to one color and a color-coded map was created. Figures 16 and 18 show the obtained ellipses (left) and the corresponding orientation map (color-coded map on the right) for each section in Fig. 5a, b respectively. Figures are not in scale for better visualization. Each image has 121 ellipses and the ellipse image size is $42 \times 42$ pixels $(11 \times 11 \mathrm{~mm})$.

The results were also statistically analyzed. First, the mean orientation angle was calculated for each section as well as the correspondent standard deviation (STD) value. Table 3 shows these values. Second, orientation values for each section were sorted and then the values were plotted. Figures 17a and 19a show these plots for the results reported in Figs. 16 and 18, respectively. Last, all possible orientation values were dived into 18 classes and each measured angle was classed into its specific class. For instance, if the fiber orientation measured for a point was $5^{\circ}$, it would be classed into class 1 (class 1 includes angles from $0^{\circ}$ to $9^{\circ}$, class 2 includes angles from $10^{\circ}$ to $19^{\circ}$, and so on). Figures $17 \mathrm{~b}$ and $19 \mathrm{~b}$ show a bar chart where the number of samples of each one of the 18 classes are shown for the results reported in Figs. 16 and 18, respectively.

By observing the results reported in Table 3 and in Figs. 17 and 19, one can easily conclude that the fiber orientation on the surface of a ROS sample is well distributed,

Table 3 Statistical results from ROS point-by-point inspection

\begin{tabular}{lll}
\hline & Front section & Back section \\
\hline Mean $\left(^{\circ}\right)$ & 93 & 82 \\
$\operatorname{STD}\left(^{\circ}\right)$ & 58.1 & 59.3 \\
\hline
\end{tabular}




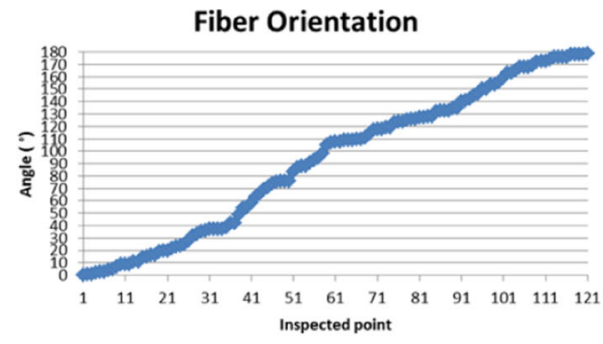

a

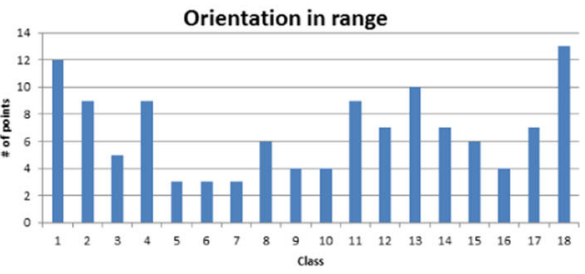

b

Fig. 17 Statical analyses of point-by-point inspection —-front section. Statistical results of point-by-point inspection of the front section of sample B61. a Plot of 121 sorted orientation values and $\mathbf{b}$ bar chart of the number of points per class

which confers (at least on its surface) a quasi-isotropic behavior to the sample. Such analysis could be extended to the other "layers" of the ROS sample confirming that the random oriented strands give a quasi-isotropic behavior to the entire sample.

\section{Line results}

The goal of the line approach is to develop a method that could estimate the fiber orientation on the surface of a ROS sample using a line heating region, that in this case was obtained with a flying laser spot inspection. ANNs were chosen because they have the capability of estimating or approximating functions that can depend on a large number of inputs and are generally unknown, which is the present case. If one observes the result obtained with the PCT, PPT, and DTT application on the reconstructed pseudo-static sequence (see Fig. 4), it can easily observe that there is information linked to the fiber orientation on the edges of the line envelope. However, a relation between this information and the actual fiber orientation is far from obvious. Thus, we propose to use an ANN.

The first step in order to employ an ANN to approximate this problem is to structure the network. In the "Methodology" section, it is summarized the ANN used in this work. Next, the network must be trained, i.e., the network must learn how to classify a sample. In order to train an ANN, a set of samples for which the classification of each sample is known beforehand must be created. In order to create a training dataset, 49 points were previously inspected by PTE on the same regions (49 on each region) where each flying laser spot inspection would be later conducted. The same approach used for the PTE was employed to assess the fiber orientation of a single point using a static laser spot heating source. Thus, 588 PTE inspections, on 12 lines from 3 different samples, were performed in order to create a database with known orientations that were used as ground truth. From this database, 412 samples were used to train the net-

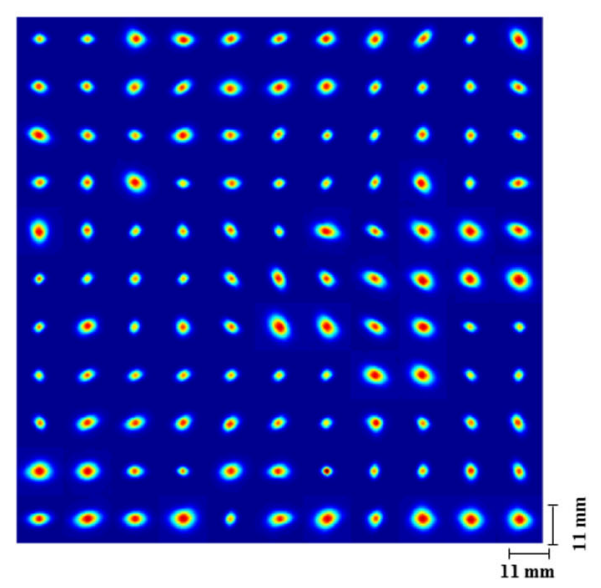

a

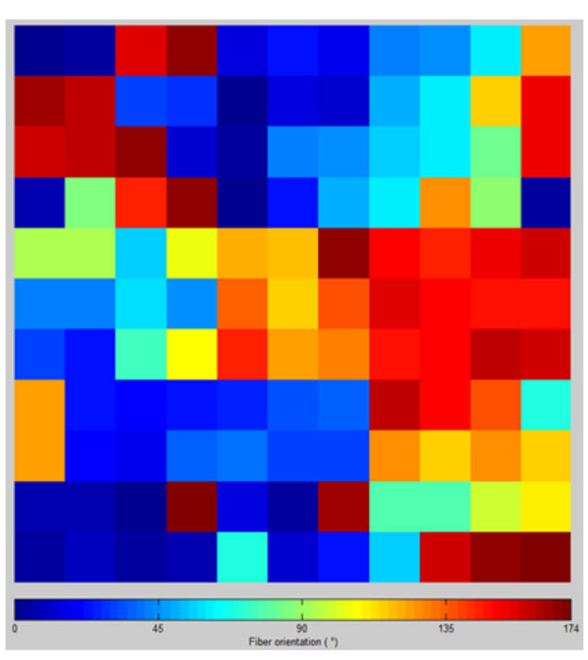

b

Fig. 18 Point-by-point inspection results-back section. Result of point-by-point inspection of the back section of sample B61. a 121 ellipses obtained and $\mathbf{b}$ respective color-coded orientation map. The actual size of the inspected region is $50 \times 50 \mathrm{~mm}^{2}$ 


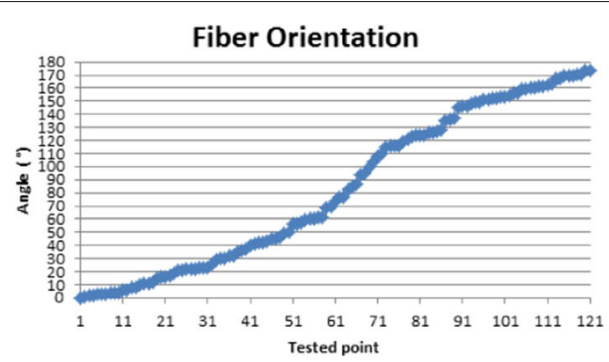

a

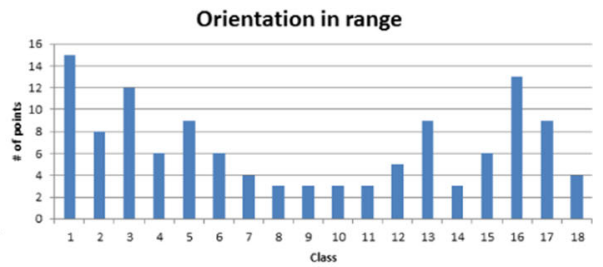

b

Fig. 19 Statical analyses of point-by-point inspection—back section. Statistical result of point-by-point inspection of the back section of sample B61. a Plot of 121 sorted orientation values and $\mathbf{b}$ bar chart of the number of points per class

work. They were presented to the network as well as their respective classes obtained from the static single-point inspections. During the training process, the network adjusts itself (its internal weights) in order to recognize the input samples presented during training into their classes with an acceptable error. From the other 176 samples, half were used for validation purposes. The other half was not presented to the network during training process and is used in order to test the network after training.

The pseudo-static sequences were processed with three different techniques: PCT, PPT, and DTT. Thus, the training, validation, and testing processing of the network was performed three times. Results were compared in order to select the best infrared image processing technique suitable for this specific problem. However, before the technique was applied on ROS samples, we tried it on the laminate case. Recognizing the fiber orientation on the surface of a laminate sample is a trivial task and the results obtained with the ANN for all three stages, i.e., training, validating, and testing, should be $100 \%$. And, in fact, we got $100 \%$ for all three infrared image processing techniques tested. After this previous step, the technique was applied on ROS samples where it would be much more difficult for the ANN to assess the fiber orientation. Results for the ROS case are presented in Table 4.

For the two techniques that presented better results, i.e., PCT and PPT, we present in Fig. 20 a comparison of results for classification of the same region. The inspected region is $100 \times 50 \mathrm{~mm}$ divided 11 lines spaced of $2 \mathrm{~mm}$ from each other and each line was divided in 49 samples. Results are color-coded according to class assigned to the

Table 4 ANN classification rates for 512 hidden neurons

\begin{tabular}{lllll}
\hline Input image & Total (\%) & Training (\%) & Validation (\%) & Test (\%) \\
\hline PCT & 84.9 & 91.3 & 68.2 & 71.6 \\
PTT & 81.1 & 88.6 & 55.7 & 71.6 \\
DTT & 76.4 & 80.6 & 61.4 & 71.6 \\
\hline
\end{tabular}

sample at the ANN's output. The results are quite different. Nevertheless, they are within the results that we could expect with a test accuracy of $71.6 \%$.

As it can be observed in Fig. 21, results obtained from PCT and PPT appear to be quite different. However, it can be observed that the differences usually occurred between neighboring classes, and at this early stage of the algorithm, it is acceptable since each class covers $45^{\circ}\left( \pm 22^{\circ}\right)$ and a sample which is placed near the border of one class could easily be misclassified as the neighbor class. For example, a sample which has fibers oriented at $20^{\circ}$ could

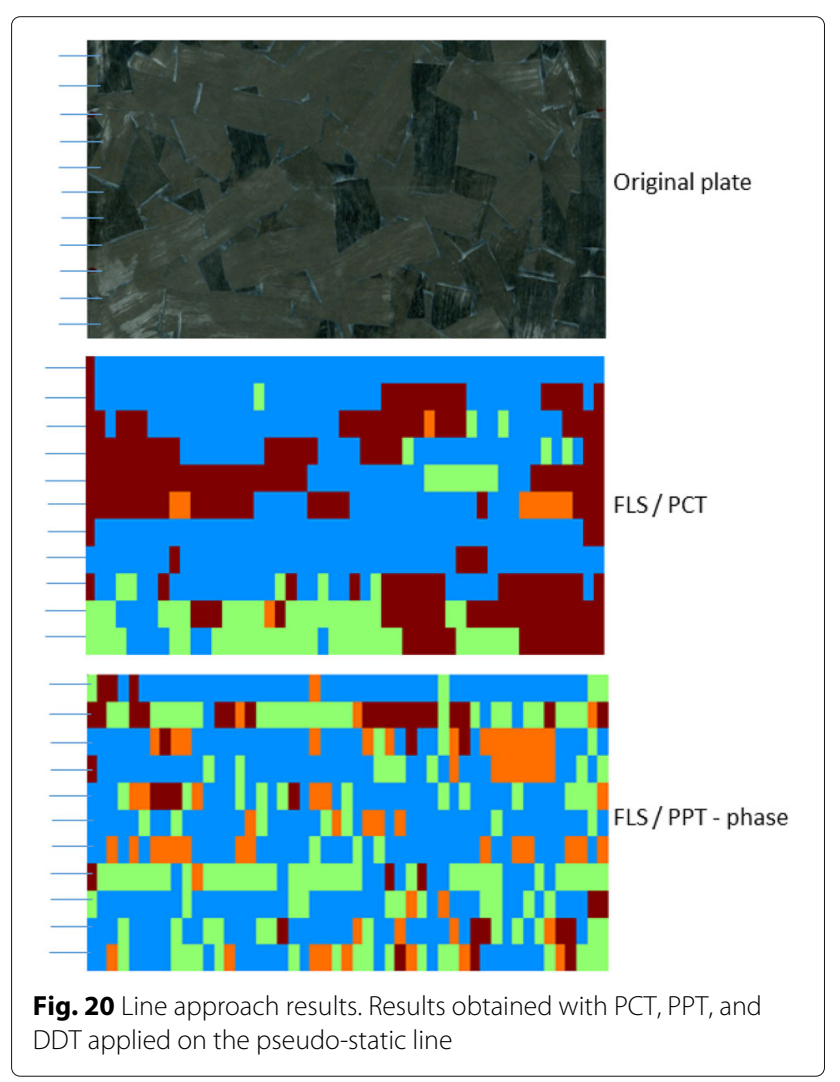



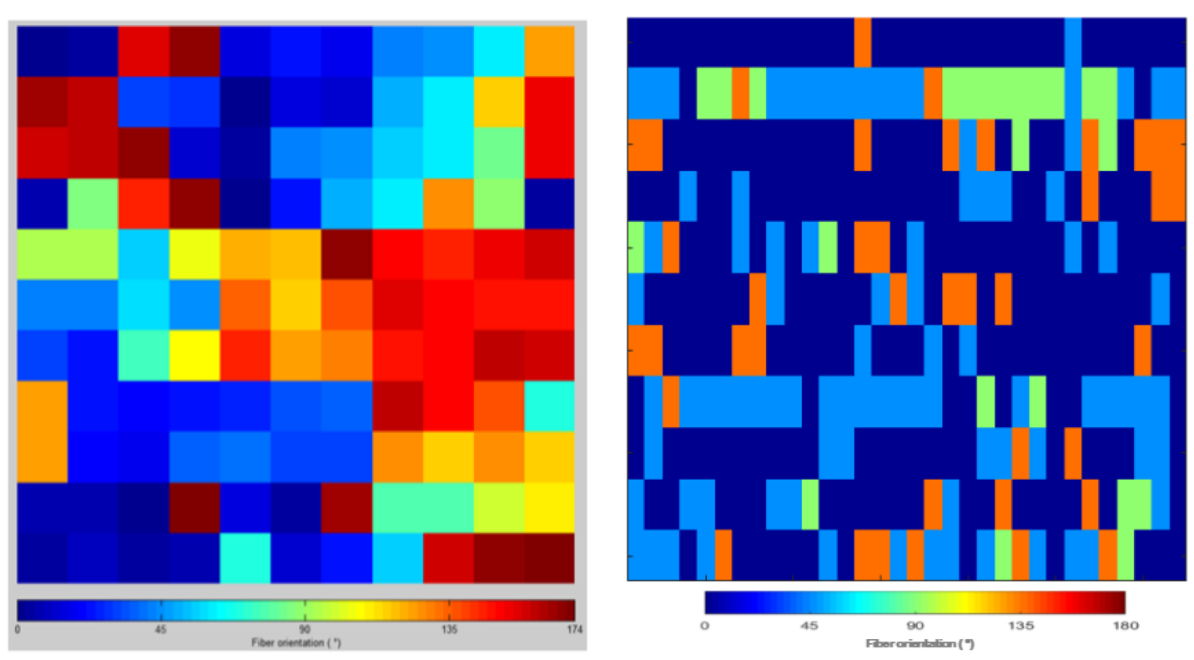

Fig. 21 Point-by-point and line approach result comparison. The same color coding used in Fig. 18b was used here. Results obtained with the line approach from the same region reported in Fig. 18b. Results calculated with the ANN for the FLS inspection are color-coded according to the target classes

be classified in class 1 (centered at $0^{\circ}$ ) by PCT and classified in class 2 (centered at $45^{\circ}$ ) by PPT since each class covers $\pm 22^{\circ}$ and the sample originally oriented at $20^{\circ}$.

Also, the number of classes was limited to four in order to develop a first solution that could be improved later. In the current state of this research, each class is $45^{\circ}$ wide. However, if six or nine classes were used instead of four $\left(30^{\circ}\right.$ or $20^{\circ}$ wide respectively instead of $\left.45^{\circ}\right)$, the performance of the network would be severely affected in the current state of this solution. Generally, the classification rate in the testing dataset decreased to 30 and $25 \%$, respectively. Nevertheless, these first results obtained with four classes are very promising.

Other configurations (number of hidden neurons) were tested for the ANN. However, the obtained results were not satisfactory. These results are presented in Table 5 . With fewer hidden neurons, the results were even worse.

\section{Comparison}

Additionally, the $50 \times 50$-mm region (Fig. 5 b) that was inspected with the static spot heating PTE technique reported in Fig. 18b was inspected with the line approach. These results are displayed in Fig. 21 and can be directly compared to the one showed in Fig. 18b. The inspected

Table 5 ANN classification rates for 256 hidden neurons

\begin{tabular}{lllll}
\hline Input image & Total (\%) & Training (\%) & Validation (\%) & Test (\%) \\
\hline PCT & 75.7 & 80.6 & 65.9 & 62.5 \\
PTT & 74.5 & 78.2 & 65.9 & 65.9 \\
DTT & 85.9 & 95.1 & 65.9 & 62.5 \\
\hline
\end{tabular}

region is depicted in Fig. 5b. The result of inspection of the region with the point-by-point PTE inspection where 121 points where inspected $(11 \times 11$ points $)$ is showed in Fig. 18b. Results are presented and color-coded in a range of $0^{\circ}$ to $180^{\circ}$. Figure 21 shows the inspection results of the same region for both the point-by-point PTE inspection and the line (FLS/ANN) approach. In this last case, 11 lines with 32 samples on each line are reported, i.e., 11 line inspections were performed with the FLS technique. Results are displayed color-coded according to the 0 to 180 range. The image processing technique used in this case was the PPT. It must be remembered here that each classification result obtained with the line approach covers a region with $45^{\circ}$ while the point-bypoint of the given classification is in the $1^{\circ}$ range. The best improvement obtained with the line approach is the inspection speed. While the PTE inspection of a single line showed in Fig. 5b took around $25 \mathrm{~min}$, the line inspection presented in Fig. 21 was performed and processed in under 30 s, i.e., 50 times less. This is an important factor when considering an approach to be used in the industry.

\section{Conclusions}

In this work, it was presented two approaches for fiber orientation assessment on ROS materials based on infrared thermography. The first one uses the classical PTE method and a point-by-point inspection procedure to evaluate the fiber orientation over an ROS region since the fibers are randomly oriented. The second approach is inspired in the PTE method. It uses a flying laser spot inspection procedure and classifies the samples using an artificial neural network. 
For the PTE approach, inspections were performed on single strands since each strand has its own fiber orientation. Ultimately, a matrix of several points on the surface of a flat ROS sample was inspected in order to estimate "how random" fiber orientation is on the sample's surface. An "orientation map" was created and statistical analysis of the results (Table 3 ) showed that the fiber orientation on a ROS sample is well distributed, which confirms the quasi-isotropic behavior of ROS samples.

However, an inspection approach based on a matrix of several points obtained with PTE of a ROS sample is very time consuming. The inspection of 121 points (Fig. 5b) presented in this paper took about $2 \mathrm{~h}$. Thus, we proposed to use the line approach described here to inspect a broader region at once. The same experiments which last for $2 \mathrm{~h}$ in the PTE case were performed in less than $6 \mathrm{~min}$ using the line approach.

Our proposed line approach uses a flying laser spot (FLS) to heat the sample over a line region. Then, the thermal sequence is rearranged and processed with three different image processing techniques: PCT, PPT, and DTT. From these processed images, features are extracted and an ANN is used to recognize the fiber orientation linked to that sample. Training and testing results were presented in Table 4. The best technique was PCT which presented an accuracy in the training stage of 91.3 and $71.6 \%$ in the testing. This is probably because of the nature of the PCT technique which tends to project the most significant information present in the data set into the first EOF images. Even though the results obtained with the ANN are not spectacular, the inspection time of the method is the great advantage of the proposed line approach.

\section{Abbreviations}

AOl: Angle of incidence; ANN: Artificial neural network; CM: Composite materials; CW: Continuous wave; FEA: Finite element analysis; FLS: Flying laser spot; IR: Infrared; IRT: Infrared thermography; MWIR: Mid-wave infrared; NDE: Non-destructive evaluation; PTE: Pulsed thermal ellipsometry; ROS: Randomly oriented strand; STD: Standard deviation; TE: Thermal ellipsometry

\section{Acknowledgements}

The authors would like to gratefully acknowledge the support provided by the Canada Research Chair in Multipolar Infrared Vision (MiViM) and the industrial partners: Bell Helicopter Textron Canada Limited, Bombardier Inc., Pratt and Whitney Canada Corp., Avior Integrated Products Inc., Delastek Inc., and Hutchinson Inc. Canada. The authors would also like to acknowledge the support of the following agencies: FAPEMIG, Minas Gerais Research Funding Foundation; CNPq, National Council for Scientific and Technological Development, Brazil; NSERC, Natural Sciences and Engineering Research Council of Canada; FQRNT, Quebec Fund for Research on Nature and Technology; and CRIAQ, Consortium for Research and Innovation in Aerospace in Quebec.

\section{Funding}

This work was partially funded by CNPq, National Council for Scientific and Technological Development, Brazil, through the grants 166285/2015-3 and 237205/2012-2.

Availability of data and materials

Please contact author for data requests.

\section{Authors' contributions}

HF designed and performed the experiments, analyzed the data, and wrote the paper. $\mathrm{HZ}$ worked in the experimental procedure. AF processed the data. FM assisted in the discussion of the results and development of the computational model. LHI assisted in the paper writing/revision and development of the computational model. $\mathrm{Cl}$-C is the research co-supervisor. $\mathrm{XM}$ is the research supervisor. The manuscript was discussed by all the coauthors. All authors read and approved the final manuscript.

\section{Competing interests}

The authors declare that they have no competing interests.

\section{Publisher's Note}

Springer Nature remains neutral with regard to jurisdictional claims in published maps and institutional affiliations.

\section{Author details}

${ }^{1}$ School of Computer Science, Federal University of Uberlandia, 2121 Joao Naves de Avila Avenue, Uberlandia 38408-100, Brazil. ${ }^{2}$ Department of Electrical and Computer Engineering, Laval University, 1065 de la Medicene, Quebec G1V 0A6, Canada. ${ }^{3}$ Department of Mechanical Engineering, Federal University of Uberlandia, 2121 Joao Naves de Avila Avenue, Uberlandia 38408-100, Brazil. ${ }^{4}$ State University of Minas Gerais, Vereador Geraldo Moises da Silva Street, Ituiutaba 38302-192, Brazil. ${ }^{5}$ Federal Institute of Santa Catarina, 830 Getulio Vargas Street, 89251-000, Jaragua do Sul Brazil.

Received: 2 March 2018 Accepted: 11 June 2018

Published online: 04 July 2018

\section{References}

1. Zhang H, Sfarra S, Sarasini F, Ibarra-Castanedo C, Perilli S, Fernandes H, Duan Y, Peeters J, Avdelidis NP, Maldague X (2018) Optical and mechanical excitation thermography for impact response in basalt-carbon hybrid fiber-reinforced composite laminates. IEEE Trans Ind Inform 14(2):514-522. https://doi.org/10.1109/TII.2017.2744179

2. Liu B, Zhang H, Fernandes H, Maldague X (2016) Experimental evaluation of pulsed thermography, lock-in thermography and vibrothermography on foreign object defect (FOD) in CFRP. Sensors 16(5). https://doi.org/10. 3390/s16050743. http://www.mdpi.com/1424-8220/16/5/743

3. Fernandes $\mathrm{H}$, Zhang $\mathrm{H}$, Figueiredo A, Ibarra-Castanedo C, Guimarares $\mathrm{G}$, Maldague X (2016) Carbon fiber composites inspection and defect characterization using active infrared thermography: numerical simulations and experimental results. Appl Opt 55(34):46-53. https://doi. org/10.1364/AO.55.000D46

4. Yousefi B, Sfarra S, Castanedo Cl, Maldague XP (2017) Comparative analysis on thermal non-destructive testing imagery applying candid covariance-free incremental principal component thermography (ccipct). Infrared Phys Technol 85:163-169

5. Maldague X (2001) Theory and practice of infrared technology for nondestructive testing. 1st edn. Wiley-Interscience, New York. p684

6. Fernandes HC, Zhang H, Ibarra-Castanedo C, Maldague X (2017) Artificial neural networks and infrared thermography for fiber orientation assessment. In: 2017 Brazilian Conference on Intelligent Systems (BRACIS). pp 210-215. https://doi.org/10.1109/BRACIS.2017.59

7. Senarmont MHD (1848) Mémoire sur la conductivité des substances cristallisées pour la chaleur: second mémoire. In: Annales de Chimie Physique Vol. 3. pp 179-211

8. Krapez JC (1994) Thermal ellipsometry applied to the evaluation of fibre orientation in composite. Tiré à part- Office national d'études et de recherches aerospatiales (171),26

9. Aindow JD, Markham ME, Puttick KE, Rider JG, Rudman MR (1986) Fibre orientation detection in injection-moulded carbon fibre reinforced components by thermography and ultrasonics. NDT\&E Int 19(1):24-29

10. Karpen W, Wu D, Busse G (1999) A theoretical model for the measurement of fiber orientation with thermal waves. Res Nondestruct Eval 11(4):179-197

11. Fernandes $H$, Zhang H, Maldague $X$ (2015) An active infrared thermography method for fiber orientation assessment of fiber-reinforced composite materials. Infrared Phys Technol 72:286-292. https://doi.org/10.1016/j.infrared.2015.07.021 
12. Yang ST, Matthews MJ, Elhadj S, Cooke D, Guss GM, Draggoo VG, Wegner PJ (2010) Comparing the use of mid-infrared versus far-infrared lasers for mitigating damage growth on fused silica. Appl Opt 49(14):2606-2616

13. Otsu N (1979) A threshold selection method from gray-level histograms. In: International Conference on Systems, Man, and Cybernetics (SMC). IEEE Vol. 9. pp 62-66

14. Fernandes $\mathrm{H}$, Zhang H, Ibarra-Castanedo C, Maldague X (2015) Fiber orientation assessment on randomly-oriented strand composites by means of infrared thermography. Compos Sci Technol 121:25-33. https:// doi.org/10.1016/j.compscitech.2015.10.015

15. Gruss C, Balageas D (1992) Theoretical and experimental applications of the flying spot camera. In: Proceedings of the QIRT 92. pp 19-24

16. Ibarra-Castanedo C, Servais P, Ziadi A, Klein M, Maldague X (2014) RITA-robotized inspection by thermography and advanced processing for the inspection of aeronautical components. In: Balageas D (ed). The 12th International Conference on Quantitative InfraRed Thermography, 7-11 July, Bordeaux, France

17. Rajic N (2002) Principal component thermography for flaw contrast enhancement and flaw depth characterisation in composite structures. Compos Struct 58(4):521-528

18. Maldague X, Marinetti S (1996) Pulse phase thermography. J Appl Phys 79(5):2694-2698

19. Vavilov VP, Maldague XP (1992) Dynamic thermal tomography: new promise in the ir thermography of solids Vol. 1682. pp 194-206. https:// doi.org/10.1117/12.58536

20. Egmont-Petersen M, Ridder Dd, Handels H (2002) Image processing with neural networks - a review. Pattern Recognit 35(10):2279-2301

21. Darabi A, Maldague X (2002) Neural network based defect detection and depth estimation in tnde. NDT\& E Int 35(3):165-175. https://doi.org/10. 1016/S0963-8695(01)00041-X

22. Benítez HD, Loaiza H, Caicedo E, Ibarra-Castanedo C, Bendada A, Maldague X (2009) Defect characterization in infrared non-destructive testing with learning machines. NDT\& E Int 42(7):630-643. https://doi. org/10.1016/j.ndteint.2009.05.004

23. Fernandes HC, Maldague XPV (2014) Fiber orientation assessment on surface and beneath surface of carbon fiber reinforced composites using active infrared thermography. In: Proc. SPIE 9105, Thermosense: Thermal Infrared Applications XXXVI, Vol. 1905. International Society for Optics and Photonics, pp. 91050-910509. SPIE, Bellingham. https://doi.org/10.1117/ 12.2053480

24. Ageorges C, Ye L, Mai Y-W, Hou M (1998) Characteristics of resistance welding of lap shear coupons. Part I: Heat transfer. Composites Part A: Applied Science and Manufacturing 29(8):899-909

\section{Submit your manuscript to a SpringerOpen ${ }^{\circ}$ journal and benefit from:}

- Convenient online submission

- Rigorous peer review

- Open access: articles freely available online

- High visibility within the field

- Retaining the copyright to your article

Submit your next manuscript at $\$$ springeropen.com 